\title{
Assessing efficacy and safety of stereotactic body radiation therapy for oligometastatic non-small cell lung cancer with epidermal growth factor receptor (EGFR) wild type
}

\author{
Xiaolong Hu ${ }^{1}$, Hongqi $\mathrm{Li}^{2}$, Hefei Liu ${ }^{2}$, Zhifei Liu ${ }^{2}$, Tingyi Xia ${ }^{2}$, Jianchun Zhang ${ }^{1}$, Yingjie Wang ${ }^{2}$ \\ ${ }^{1}$ Department of Radiation Oncology, Beijing Geriatric Hospital, Beijing, China; ${ }^{2}$ Department of Radiation Oncology, Air Force General Hospital, \\ Beijing, China \\ Contributions: (I) Conception and design: Y Wang, X Hu; (II) Administrative support: J Zhang; (III) Provision of study materials or patients: H Li; (IV) \\ Collection and assembly of data: H Liu; (V) Data analysis and interpretation: X Hu, T Xia; (VI) Manuscript writing: All authors; (VII) Final approval \\ of manuscript: All authors. \\ Correspondence to: Yingjie Wang. Department of Radiation Oncology, Air Force General Hospital, Beijing, China. Email: Wangyj9999@163.com.
}

Background: Stereotactic body radiation therapy (SBRT) is an emerging therapy for oligometastatic cancer. The aim of this study was to investigate the efficacy and safety of high-dose radiotherapy for primary and oligometastatic lesions in epidermal growth factor receptor (EGFR) wild-type non-small cell lung cancer (NSCLC).

Methods: A total of 40 EGFR wild-type oligometastatic NSCLC patients (defined as $\leq 5$ metastases) treated with SBRT in our department between 2009 and 2016 were analyzed retrospectively. SBRT was delivered to the lesions with a median biologically effective dose at alpha/beta 10 (BED10) value of 102.7 Gy (range, 94.5-113.5 Gy). Primary endpoints including progression-free survival (PFS) and overall survival (OS) were estimated with the Kaplan-Meier method. Factors potentially affecting OS and PFS were evaluated by univariate and multivariate Cox-regression analyses.

Results: After a median follow-up of 39 months, the median OS observed in this study was 40 months (95\% CI: 32.562-47.438 months). One-, 2-, and 3-year OS rates were 100.0\%, 72.5\%, and 62.5\% respectively. Twenty-nine patients (72.5\%) succumbed to tumor burden and median PFS was 13 months (range, 10.68715.313 months). One-, 2-, and 3-year PFS rates were 65.0\%, 10.0\%, and 0\% respectively. Multivariate analysis suggested Eastern Cooperative Oncology Group performance status (ECOG PS) $<2$ and high-dose radiation regimens were independent prognostic factors of longer $\mathrm{OS}(\mathrm{P}<0.001$ and 0.049 , respectively), and patients receiving radiotherapy with BED $10 \geq 100$ Gy showed a better PFS than those undergoing low dose $(\mathrm{P}=0.047)$. There were no patients of CTCAE v 5.0 grade 4-5 toxicity or treatment-related deaths. Grade 3 toxicity occurred in $2(5.0 \%)$ patients and 36 (90.0\%) patients experienced grade $1-2$ adverse reactions.

Conclusions: The current study suggested systemic chemotherapy combined with SBRT for pulmonary and metastatic lesions was feasible and tolerable to improve outcomes of EGFR wild-type oligometastatic NSCLC patients.

Keywords: Epidermal growth factor receptor (EGFR); oligometastasis; non-small cell lung cancer (NSCLC); stereotactic body radiation therapy (SBRT)

Submitted Aug 21, 2020. Accepted for publication Nov 16, 2020.

doi: $10.21037 /$ tcr-20-2772

View this article at: http://dx.doi.org/10.21037/tcr-20-2772 


\section{Introduction}

Lung cancer is the leading cause of cancer-associated death worldwide (1), among which non-small cell lung cancer (NSCLC) represents about $85 \%$ of all cases $(2,3)$. More than half of the NSCLC patients initially diagnosed with distant metastases (4). Mehta et al. reported that $(5,6)$ patients with metastatic NSCLC could remain relatively stabilization and not be widely disseminated. In 1995, Hellman and Weichsel (7) proposed the concept of "oligometastasis" for the first time, which was used to describe the state between localized and widespread metastatic disease. Recently, oligometastasis was defined as limited metastasis with a maximum 5 clinically detectable lesions restricted to few organs (usually 1-3) $(8,9)$. Oligometastasis may possibly occur at early stage of tumor dissemination and correlate with the indolent biological behavior of tumor progression. The presence of oligometastatic disease may provide a novel opportunity to improve the prognosis of epidermal growth factor receptor (EGFR) wild-type NSCLC patients through local ablative therapies (LAT) such as surgery, radiotherapy, radiofrequency ablation or cryoablation (10).

Platinum-based doublets chemotherapy continues to be the standard of care for patients with stage IV NSCLC, which carry a poor prognosis with median survival times of less than 1 year (11). Recently, targeted therapy and immunotherapy have showed significant advances in treatment of NSCLC, including identification of EGFR mutations and anaplastic lymphoma kinase (ALK) rearrangement (12). A previous study has suggested that combined LAT with EGFR tyrosine kinase inhibitors (TKIs) showed a significantly longer progression-free survival (PFS) and overall survival (OS) than TKIs alone in NSCLC with oligometastatic liver metastases cohort (13). However, for patients with EGFR wild-type oligometastatic NSCLC, the optimal treatment remains controversial. Sheu et al. (14) reported that patients with stage IV NSCLC receiving at least 2 cycles of chemotherapy before comprehensive local therapy had a significantly better survival prognosis as compared to patients who received only monotherapy and the subgroup with lower ECOG PS $(\leq 1)$ predicted improved OS (14). Moreover, it has been shown that stereotactic body radiation therapy (SBRT) for pulmonary lesions in oligometastatic NSCLC was a safe and feasible procedure (15).

However, most of the published literature focused on the effect of SBRT on the pulmonary foci. The aim of the present study was to investigate the efficacy and adverse effects of high-dose radiotherapy treating for primary tumors and all metastatic lesions of oligometastatic NSCLC patients with EGFR wild-type. We present the following article in accordance with the STROBE reporting checklist (available at http://dx.doi.org/10.21037/tcr-20-2772).

\section{Methods}

\section{General clinical data}

From February 2009 to October 2016, we enrolled 1,238 lung cancer patients who were treated with radiotherapy at the Radiation Therapy Department of the Air Force General Hospital. Among them, a total of 40 patients harboring EGFR wild-type oligometastatic NSCLC were treated with SBRT for all pulmonary and metastatic lesions (baseline patient characteristics are depicted in Table 1). This retrospective study was conducted in accordance with the Declaration of Helsinki (as revised in 2013) and approved by the Ethics Committee of Air Force General Hospital (NO.: BJLNYY-\#2020009). The patients were included in this study only after obtaining orally or written informed consent.

\section{Inclusion and exclusion criterions}

The inclusion criteria were listed as follows: (I) the diagnosis of NSCLC confirmed by histological/pathological examination EGFR wild-type genotype confirmed by genetic testing (amplification refractory mutation systemPCR method); (II) oligometastasis (total metastases within five lesions) confirmed by comprehensive imaging examination (included but not limited to brain magnetic resonance imaging $(\mathrm{MRI})+$ whole-body positron emission tomography/computed tomography (PET/CT) or brain MRI + thoracic/abdominal/pelvic CT, and bone scan when necessary); (III) after systemic therapy, the primary tumor and all metastases maintaining stable (no observed progression or new metastasis for at least one month); (IV) age >18 years old; (V) before the onset of SBRT, bone marrow hematopoietic function, lung function, cardiac function, and liver function reserve remaining within the normal range; (VI) all lesions suited for high-dose radiation therapy; (VII) for patients receiving chemotherapy, sequential radiotherapy following the end of chemotherapy (If there was central nervous system (CNS) metastases, local radiotherapy for intracranial lesions would be performed preferentially). 
Table 1 Baseline patient characteristics

\begin{tabular}{|c|c|}
\hline Characteristics & N (\%) \\
\hline \multicolumn{2}{|l|}{ Gender } \\
\hline Female & $23(57.5)$ \\
\hline Male & $17(42.5)$ \\
\hline \multicolumn{2}{|l|}{ Age (years) } \\
\hline$<65$ & $27(67.5)$ \\
\hline$\geq 65$ & $13(32.5)$ \\
\hline \multicolumn{2}{|l|}{ Histology } \\
\hline Non-adenocarcinoma & $9(22.5)$ \\
\hline Adenocarcinoma & $29(72.5)$ \\
\hline NSCLC & $2(5.0)$ \\
\hline \multicolumn{2}{|l|}{ Smoking status } \\
\hline No & $28(70.0)$ \\
\hline Yes & $12(30.0)$ \\
\hline \multicolumn{2}{|l|}{ Smoking index } \\
\hline$<800$ & $24(60.0)$ \\
\hline$\geq 800$ & $16(40.0)$ \\
\hline \multicolumn{2}{|l|}{ ECOG PS } \\
\hline$<2$ & $30(75.0)$ \\
\hline$\geq 2$ & $10(25.0)$ \\
\hline \multicolumn{2}{|l|}{$\mathrm{N}$ stage } \\
\hline N0-N1 & $14(35.0)$ \\
\hline N2 & $17(42.5)$ \\
\hline N3 & $9(22.5)$ \\
\hline \multicolumn{2}{|l|}{ CNS metastases } \\
\hline No & $27(67.5)$ \\
\hline Yes & $13(32.5)$ \\
\hline \multicolumn{2}{|l|}{ No. of metastases organs } \\
\hline 1 & $32(80.0)$ \\
\hline$\geq 2$ & $8(20.0)$ \\
\hline \multicolumn{2}{|l|}{ No. of metastases } \\
\hline 1 & $20(50.0)$ \\
\hline 2 & $12(30.0)$ \\
\hline $3-5$ & $8(20.0)$ \\
\hline
\end{tabular}

Table 1 (continued)
Table 1 (continued)

\begin{tabular}{lc}
\hline Characteristics & $\mathrm{N}(\%)$ \\
\hline $\mathrm{BED}_{10} \geq 100$ Gy & $21(52.5)$ \\
No & $19(47.5)$ \\
Yes & \\
PET-CT & $1(2.5)$ \\
No & $39(97.5)$ \\
Yes & \\
\hline
\end{tabular}

Smoking index: number of cigarettes smoked per day $\times$ years of smoking. NSCLC, non-small cell lung cancer; ECOG PS, Eastern Cooperative Oncology Group performance status; CNS, central nervous system; BED, biological effective dose; PET-CT, positron emission tomography-computed tomography.

The exclusion criterions were set as follows: (I) patients with other primary tumor simultaneously; (II) patients who had undergone radiation therapy for a primary or metastatic lesion previously; (III) pregnant or lactating women.

The clinical characteristics of included patents were presented in Table 1.

\section{Radiation dose mode}

Forty oligometastatic NSCLC patients with a total of 210 lesions were treated with SBRT and median $\mathrm{BED}_{10}$ value was 102.7 Gy (range, 94.5-113.5 Gy) delivered to lesions. The characteristics of lesions and radiation dose were presented in Table 2.

\section{Evaluation of adverse reactions}

(I) Acute reactions: acute adverse effect was defined as toxicity occurring from the start of radical radiotherapy for all lesions to 3 months after the end of radiotherapy.

(II) Late toxicities: adverse reactions occurring after 3 months of radical radiotherapy. Acute and late adverse effect were graded according to the Common Terminology Criteria for Adverse Events version 5.0 (CTCAE v5.0) standard as follows: Grade 1, asymptomatic or mild symptoms: clinical or diagnostic observations only; Grade 2, moderate symptoms: limiting age-appropriate instrumental daily activities; Grade 3, severe or medically significant symptoms 
Table 2 Radiation dose segmentation mode

\begin{tabular}{ll}
\hline Sites of disease & Treatment regimen \\
\hline TOMO Therapy Hi·Art & \\
Lung/lymph nodes & Dt 60-75 Gy, 10-20 f \\
Bone/adrenal & Dt 60-70 Gy, 10-20 f \\
Thoracic/lumbar vertebra & Dt 30-60 Gy, 10-20 f \\
Brian & Dt 60-70 Gy, 10-20 f \\
Spinal cord & Dt 40 Gy,20f \\
Gamma-knife radiosurgery & \\
Lung/bone/adrenal/lymph & $70 \%$ isodose line Dt 70-78.4 Gy, \\
nodes & $10-14 \mathrm{f}$ \\
Liver & $70 \%$ isodose line Dt 84-98 Gy, \\
& $10-12 \mathrm{f}$ \\
\hline
\end{tabular}

Dt, dose of target.

but not immediately life-threatening: limiting selfcare for daily living activities; Grade 4, life-threatening reactions: urgent intervention indicated; Grade 5, death related to adverse effect (16). Reported acute toxicities included radiation pneumonia (RP), radiation dermatitis (RD), anemia, leukopenia, thrombocytopenia, esophagitis etc. Long-term toxicities included pulmonary fibrosis, pleural effusion etc.

\section{Statistical methods}

All statistical tests were performed using Statistical Package for Social Scientists (SPSS/Windows, Version 24.0, SPSS, Inc., Chicago, IL, USA). Included patients' characteristics were described by categorical variables (frequency and percentage). Survival analysis was conducted, and KaplanMeier plot was used for calculating the survival curves. Univariable and multivariable Cox proportional hazard model were used to assess prognostic factors and calculate the survival hazard ratio (HR) with $95 \%$ confidence interval (95\% CI) of PFS and OS. All prognosis-related factors were included in a multivariable Cox model, regardless of their significance level of the univariate analysis. A two-sided $\mathrm{P}$ value of $<0.05$ was considered statistically significant in all aforementioned statistical tests.

\section{Efficacy evaluation and follow-up}

A follow-up CT or MR scan was performed 1 month after the initiation of radiotherapy and then every 3 months. All included patients were followed up until death or lost to follow-up. The therapeutic effects of SBRT were evaluated according to RECIST 1.1 (Response Evaluation Criteria in Solid Tumors, version 1.1). PFS was defined as the time from SBRT initiation to the first RECIST 1.1 defined progression (increase in the diameter of primary/ metastatic tumor or the number of new lesions). OS was calculated from the date of SBRT initiation to death of any cause or censored at the last date of follow-up. Followup information was obtained by outpatient reviews or telephone conversations.

\section{Results}

\section{Treatment and survival situation}

A total of 40 patients enrolled in the study treated with SBRT for a total of 210 lesions. SBRT was delivered to the lesions with a median $\mathrm{BED}_{10}$ value of $102.7 \mathrm{~Gy}$ (range, 94.5-113.5 Gy). Among them, 19 patients received highdose radiation regimens for all pulmonary and metastatic lesions with $\mathrm{BED}_{10} \geq 100 \mathrm{~Gy}$. Irradiated metastatic sites included 66 metastatic regional lymph nodes, 10 intrapulmonary metastases, 16 intracerebral metastases, 11 intrahepatic metastases, 13 adrenal metastases, 23 metastatic lesions in thoracic vertebra, 20 metastatic lesions in lumbar vertebra and 10 other bone metastatic lesions. As of October 4, 2020, 39 of 40 patients (97.5\%) died. Among them, 29 patients $(72.5 \%)$ died of multiple organ failure due to tumor progression, 1 patient $(2.5 \%)$ died of pulmonary embolism, 2 patients $(5.0 \%)$ died of severe infectious pneumonia, 2 patients $(5.0 \%)$ died of acute myocardial infarction, and 5 patients (12.5\%) died of unknown causes. One-, 2-, and 3-year OS rates were 100.0\%, 72.5\%, 62.5\%, respectively (Figures 1,2). One-, 2-, and 3-year PFS rates were $65.0 \%, 10.0 \%$, and $0 \%$, respectively (Figures 3,4). The median OS and PFS observed in this study were 40 months (95\% CI: 32.562-47.438 months) and 13 months (95\% CI: $11.75-18.25$ months), respectively.

\section{Adverse events}

At the time of data analysis, grade 1-2 adverse reactions occurred in $90 \%$ of patients. Two patients $(5.0 \%)$ had grade 3 adverse effects, and there were no patients of grade 4-5 toxicity or treatment-related deaths according to the CTCAE v5.0 standard (as shown in Table S1). The most 


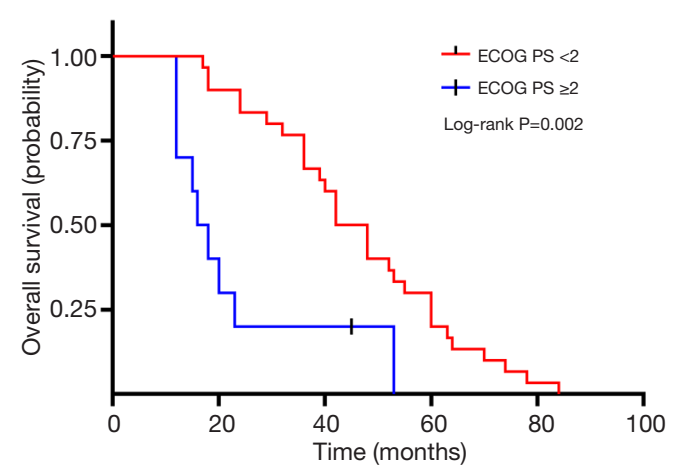

Figure 1 Kaplan-Meier plot of OS in EGFR wild-type oligometastatic NSCLC patients with ECOG PS $<2$ or $\geq 2$. OS, overall survival; EGFR, epidermal growth factor receptor; NSCLC, non-small cell lung cancer; ECOG PS, Eastern Cooperative Oncology Group performance status.

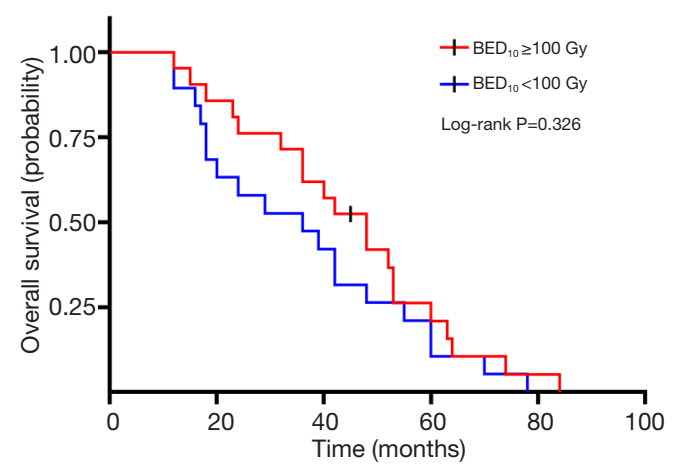

Figure 2 Kaplan-Meier plot of OS in EGFR wild-type oligometastatic NSCLC patients receiving radiotherapy with a dose regimen of $\mathrm{BED}_{10} \geq 100$ or $<100$ Gy. OS, overall survival; EGFR, epidermal growth factor receptor; NSCLC, non-small cell lung cancer.

common acute response was leukopenia $(50.0 \%, \mathrm{n}=20)$, followed by RP $(27.5 \%, \mathrm{n}=11)$ and thrombocytopenia $(25.0 \%, \mathrm{n}=10)$. Long-term adverse reactions definitely related to treatment pulmonary fibrosis $(10.0 \%, \mathrm{n}=4)$, pleural effusion $(2.5 \%, \mathrm{n}=1)$.

\section{Prognostic factors analysis}

The results of the univariate and multivariate Cox regression analysis of factors affecting OS and PFS are shown in Tables 3,4. As for clinical factors, Eastern Cooperative Oncology Group performance status (ECOG PS) were significant PFS-related prognostic factors in univariate

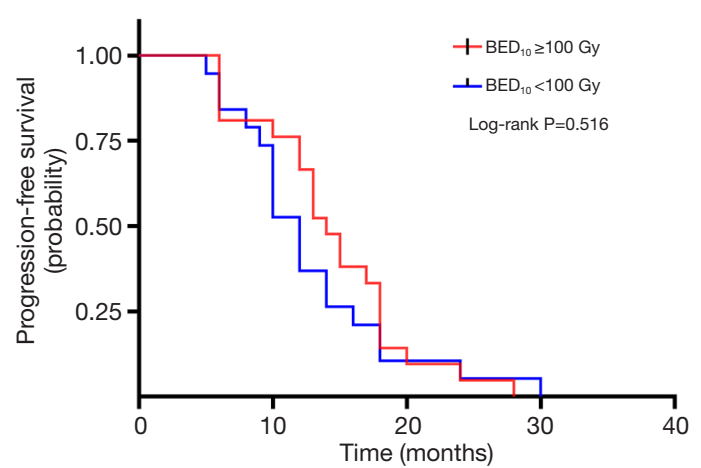

Figure 3 Kaplan-Meier plot of PFS in EGFR wild-type oligometastatic NSCLC patients receiving radiotherapy with a dose regimen of $\mathrm{BED}_{10} \geq 100$ or $<100 \mathrm{~Gy}$. PFS, progression-free survival; EGFR, epidermal growth factor receptor; NSCLC, nonsmall cell lung cancer.

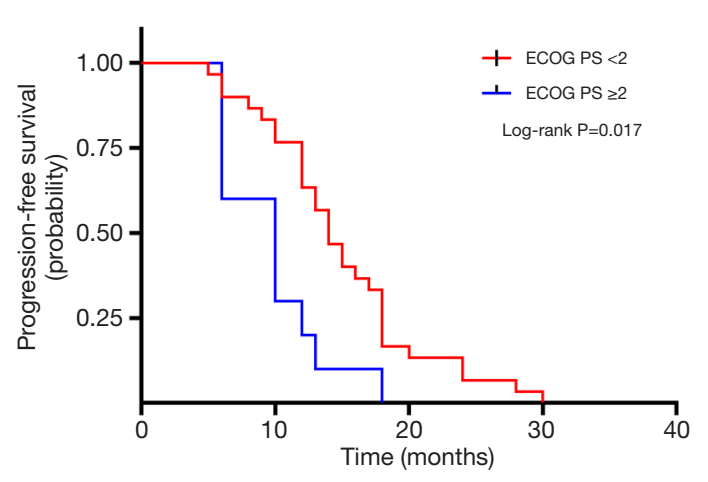

Figure 4 Kaplan-Meier plot of PFS in EGFR wild-type oligometastatic NSCLC patients with ECOG performance status $<2$ or $\geq 2$. ECOG PS, Eastern Cooperative Oncology Group performance status; PFS, progression-free survival; EGFR, epidermal growth factor receptor; NSCLC, non-small cell lung cancer.

analyses $(\mathrm{HR}=0.396,95 \% \mathrm{CI}: 0.185-0.845, \mathrm{P}=0.017)$. In multivariate analysis, $\mathrm{BED}_{10} \geq 100 \mathrm{~Gy}(\mathrm{HR}=0.374$, 95\% CI: $0.142-0.987, \mathrm{P}=0.047$ ) were significantly associated with PFS. Other factors such as smoking status, and histological type were not found to be prognostic factors for PFS. Regarding OS in univariate analyses, patients with ECOG PS $<2$ showed a better prognosis compared to $\mathrm{ECOG} \geq 2$ ( $\mathrm{HR}=0.276,95 \% \mathrm{CI}$ : $0.123-0.619, \mathrm{P}=0.002)$. In multivariate analysis, OS was most significantly influenced by ECOG PS (HR $=0.069$, 95\% CI: $0.015-0.309, \mathrm{P}<0.001)$ and radiotherapy dose. 
Table 3 Factors associated with overall survival in univariate and multivariate analyses

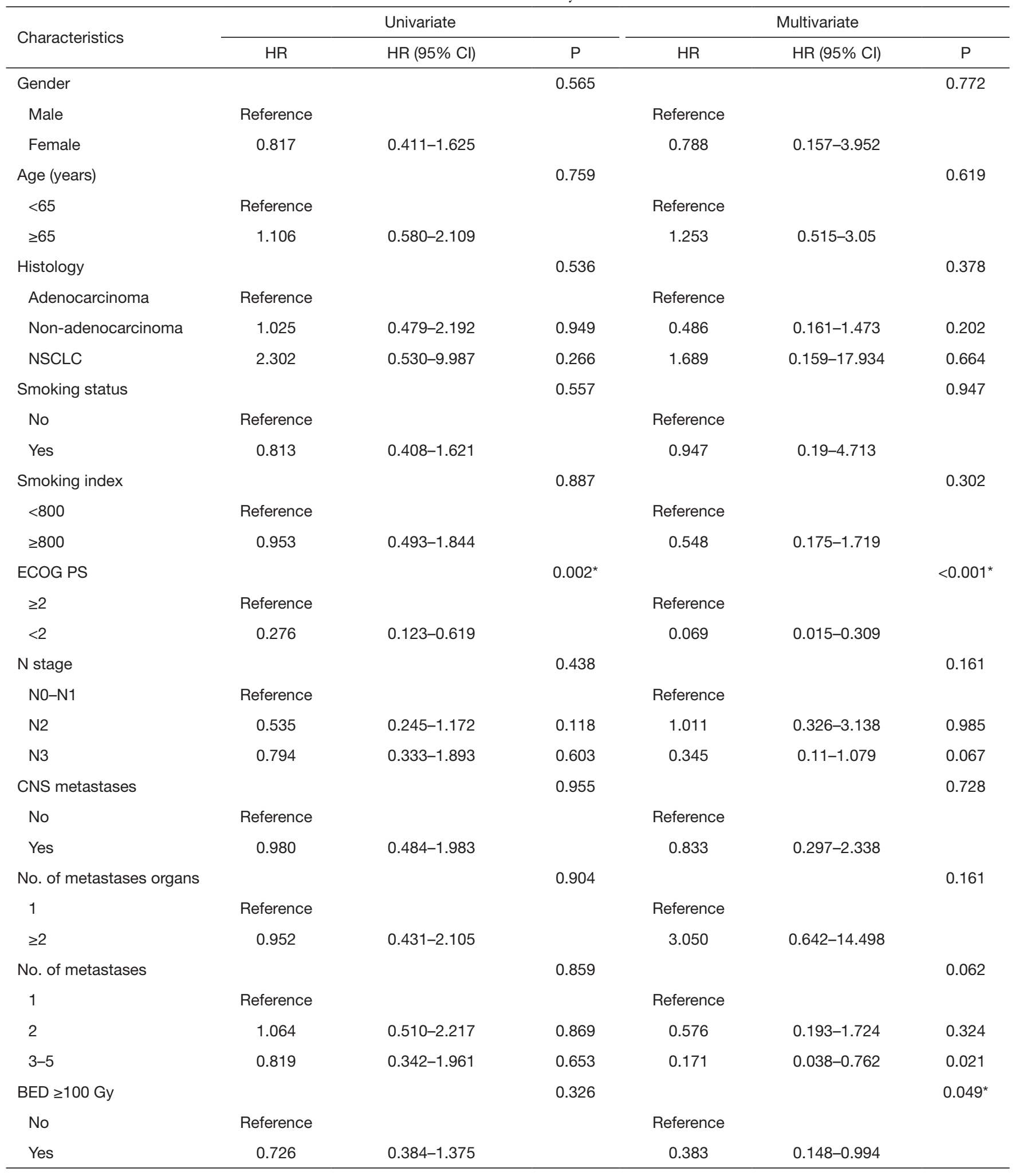

Smoking index: number of cigarettes smoked per day $\times$ years of smoking. *, statistically significant differences. HR, hazard ratio; Cl, confidence interval; NSCLC, non-small cell lung cancer; ECOG PS, Eastern Cooperative Oncology Group performance status; CNS, central nervous system; BED, biological effective dose. 
Table 4 Factors associated with progress free survival in univariate and multivariate analyses

\begin{tabular}{|c|c|c|c|c|c|c|}
\hline \multirow{2}{*}{ Characteristics } & \multicolumn{3}{|c|}{ Univariate } & \multicolumn{3}{|c|}{ Multivariate } \\
\hline & $\mathrm{HR}$ & $\mathrm{HR}(95 \% \mathrm{Cl})$ & $\mathrm{P}$ & $\mathrm{HR}$ & $\mathrm{HR}(95 \% \mathrm{Cl})$ & $\mathrm{P}$ \\
\hline Gender & & & 0.411 & & & 0.765 \\
\hline Male & 0.750 & $0.378-1.489$ & & 1.266 & $0.27-5.928$ & \\
\hline Age (years) & & & 0.154 & & & 0.182 \\
\hline$\geq 65$ & 1.623 & $0.834-3.157$ & & 1.863 & $0.747-4.642$ & \\
\hline Histology & & & 0.947 & & & 0.499 \\
\hline NSCLC & Reference & & & Reference & & \\
\hline adenocarcinoma & 1.266 & $0.272-5.884$ & 0.764 & 0.502 & $0.039-6.455$ & 0.597 \\
\hline No & Reference & & & Reference & & \\
\hline Yes & 0.759 & $0.374-1.539$ & & 0.278 & $0.046-1.667$ & \\
\hline Smoking Index & & & 0.998 & & & 0.411 \\
\hline$<800$ & Reference & & & Reference & & \\
\hline$\geq 800$ & 0.999 & $0.527-1.894$ & & 0.661 & $0.246-1.775$ & \\
\hline ECOG PS & & & $0.017^{\star}$ & & & 0.066 \\
\hline$\geq 2$ & Reference & & & Reference & & \\
\hline$<2$ & 0.396 & $0.185-0.845$ & & 0.355 & $0.117-1.071$ & \\
\hline Yes & Reference & & & Reference & & \\
\hline No & 1.027 & $0.504-2.094$ & & 0.855 & $0.299-2.449$ & \\
\hline No. of metastases organs & & & 0.824 & & & 0.769 \\
\hline 1 & Reference & & & Reference & & \\
\hline$\geq 2$ & 0.910 & $0.398-2.081$ & & 0.812 & $0.202-3.258$ & \\
\hline No. of metastases & & & 0.319 & & & 0.946 \\
\hline 1 & Reference & & & Reference & & \\
\hline 2 & 1.342 & $0.636-2.831$ & 0.440 & 1.145 & $0.451-2.907$ & 0.775 \\
\hline $3-5$ & 1.901 & $0.811-4.455$ & 0.139 & 0.998 & $0.255-3.917$ & 0.998 \\
\hline $\mathrm{BED}_{10} \geq 100$ Gy & & & 0.516 & & & $0.047^{\star}$ \\
\hline Yes & Reference & & & Reference & & \\
\hline No & 0.811 & $0.430-1.527$ & & 0.374 & $0.142-0.987$ & \\
\hline
\end{tabular}

Smoking index: number of cigarettes smoked per day $\times$ years of smoking. *, statistically significant differences. HR, hazard ratio; Cl, confidence interval; NSCLC, non-small cell lung cancer; ECOG PS, Eastern Cooperative Oncology Group performance status; CNS, central nervous system; BED, biological effective dose. 
Patients who received high-dose radiotherapy $\left(\mathrm{BED}_{10}\right.$ $\geq 100 \mathrm{~Gy}$ ) had a more favorable OS than those with $\mathrm{BED}_{10}$ $<100$ Gy (HR =0.383, 95\% CI: 0.148-0.994, P=0.049).

\section{Discussion}

Currently, the prognosis of patients with EGFR-mutant stage IV NSCLC was significantly improved with the use of TKIs. For patients with advanced NSCLC and wild-type EGFR, platinum-based doublets chemotherapy rather than TKIs has been regarded as the standard treatment during the past two decades. Despite the therapeutic benefits of combined chemotherapy, outcomes for patients with advanced NSCLC remain poor, and the median OS was only 8-10 months (17). Recent research has focused on the oligometastatic state where limited number of metastatic tumors (usually 1-5) localized in few anatomic sites, providing new opportunity in the management of advanced NSCLC with LAT. Limited tumor burden and indolent biological behavior determined oligometastatic disease remains controllable in this stage, and chemotherapy combined with local treatment (such as radiotherapy) may provide long-term remission or potential cure (10). A recent multicenter randomized phase II study demonstrated that local consolidative therapy (radiotherapy or radical surgery) with or without maintenance therapy improved PFS compared with maintenance cytotoxic treatment alone for patients with oligometastatic NSCLC (less than 3 metastases and ECOG PS $\leq 2)(18)$. Another prospective single-arm phase II trial showed that radical local treatment (surgery or radiotherapy) for synchronous oligometastatic NSCLC patients was well tolerated and associated with long-term PFS (19).

The technological advances over the past several years of SBRT have provided an emerging promising approach for such patients. Agolli et al. conducted a retrospective study that included 22 patients who were treated with SBRT. In the study, the complete response rate was $21 \%$ and the partial response (PR) rate $69 \%$. Median PFS and OS were 18 and 24 months, respectively (20). Another retrospective study indicated SBRT for oligometastatic NSCLC ( $\leq 3$ foci) resulted in long-term local control (LC), with 1-, 2-and 3 -year LC rates of $93 \%, 93 \%$ and $78 \%$, respectively (21). Rusthoven et al. reported that SBRT could improve time to progression in advanced NSCLC patients who had extracranial progression in local or distant sites after receiving first-line systemic therapy (22). Landmark clinical trials $(23,24)$ have shown that EGFR-mutant advanced
NSCLC patients had higher response rates, milder sideeffects and longer PFS when treated with EGFR-TKIs than those treated with classical platinum-based chemotherapy. Studies by Xu et al. showed that (25) LAT to all metastatic foci during first-line EGFR-TKIs treatment could significantly improve PFS and OS of patients with stage IV EGFR-mutant NSCLC. In our research, 40 patients with EGFR wild-type oligometastatic NSCLCs were treated by SBRT combined with systemic therapies. The 1-, 2-, and 3 -year OS rates were $100.0 \%, 72.5 \%, 62.5 \%$, respectively and the median OS was 40 months. Although all the enrolled patients were EGFR wild-type in our cohort, which predicted poor prognosis of NSCLC, SBRT with BED $_{10}$ $\geq 100$ Gy could provide more favorable OS and PFS. In the present study, 19 patients (47.5\%) received radiotherapy delivered to primary tumors and all metastases with a dose regimen of $\mathrm{BED}_{10} \geq 100 \mathrm{~Gy}$. Multivariate analysis showed that $\mathrm{BED}_{10} \geq 100 \mathrm{~Gy}$ was an independent prognostic factor OS (HR $=0.383,95 \%$ CI: $0.148-0.994, \mathrm{P}=0.049)$ and PFS (HR $=0.374,95 \%$ CI: 0.142-0.987, P=0.047). Lopez Guerra et al. also reported that oligometastatic NSCLC patients receiving higher dose radiotherapy to the primary tumor could be safe and more efficacious (better local-regional control and OS rates) in managing larger tumors (9).

Our study found that ECOG PS $<2$ was associated with improved prognosis in both univariate and multivariate analyses, which is consistent with previous studies by Sheu et al. (14). Favorable ECOG PS indicated that the patients can tolerate more aggressive therapy and may benefit more from SBRT. Patients with low ECOG PS frequently showed better nutritional status and immune function, which can be helpful for them to receive sequential treatment after the disease progression. In our study, the rate of patients with ECOG PS $<2$ who received subsequent therapy was $90 \%$, and this rate is $46 \%$ in patients with ECOG PS $\geq 2$. In addition, our study showed that factors such as the $\mathrm{N}$ stage, quantity of metastatic foci and location of metastases had no significant impact on PFS or OS. Thirteen cases in our cohort developed CNS metastasis from primary tumors and 5 patients of them received local radiotherapy for intracranial lesions preferentially due to severe central nervous symptoms. The median OS of patients with CNS metastases is 34.5 months. We did not find a statistically significant survival benefit for patients without CNS metastases, suggesting multiorgan or cerebral involvement were not contraindication of radical radiotherapy for patients with good PS. Nicosia et al. reported that multiple stereotactic radiosurgeries using a mono-isocentric, non- 
coplanar technique is more effective and safe for the treatment of limited brain metastases compared with wholebrain radiotherapy (26). SBRT with curative intent remains rational options for CNS metastasis in patients with favorable PS.

Widder et al. conducted a retrospective study comparing the effectiveness of pulmonary metastasectomy and SBRT. The study showed that metastasectomy as the first therapeutic option was not better than SBRT as secondline treatment (27). According to the study of Nicosia et al., SBRT is able to delay the transition of oligometastatic disease to polymetastatic disease and maintain the stable state of metastatic foci (28). Phenotypic subgroups of oligometastatic NSCLC based on clinical and biological factors may be helpful to select optimal radiotherapeutic strategy. Greco et al. developed a baseline PET/CT-based, 3-tiered categorization, potentially promoting phenotypedriven oligometastatic cancer therapy. Category 1 represents the classical oligometastatic phenotype, which can be cured with LAT before the occurrence of polymetastatic escape. Category 2 patients have an indication for consented oligometastatic radioablation and about $60 \%$ of them hold a chance of attaining cure. However, for category 3 radioablation should be performed as a component of clinical trials to address the risk of polymetastatic dissemination (29).

A retrospective study conducted by Jiang et al. which included 92 patients with EGFR-mutant NSCLC and oligometastatic or oligoprogressive liver metastases. In their cohort, patients received EGFR-TKI plus LAT had a significantly longer PFS (13.8 vs. 8.6 months) and OS (31.2 vs. 18.5 months) than those receiving only TKIs (13). Another single-institution randomized phase 2 study enrolled a total of 29 patients with stage IV NSCLC whose tumors didn't possess EGFR/ALK-targetable mutations and achieve PR or stable disease (SD) after first-line chemotherapy (30). Among them, 14 patients received SBRT plus maintenance chemotherapy and 15 patients received maintenance chemotherapy alone (PFS 9.7 vs. 3.5 months). This study showed consolidative SBRT before maintenance chemotherapy nearly tripled PFS in patients with oligometastatic NSCLC compared with chemotherapy alone. The results of our study showed that a median PFS of 13 months and a median OS of 40 months, obtained from SBRT after first-line systemic therapy. Taken together results of the above studies, definitive radiotherapy delivering to the primary tumor and all metastases on the basis of systemic chemotherapy could delay the transition to the polymetastatic disease, reduce the tumor load, and significantly improve PFS.

Despite evidence supporting SBRT for EGFR wild-type oligometastatic NSCLC, our results need to be interpreted with caution given several limitations. One limitation was that this single-institution retrospective study has inevitable selection bias for patients receiving higher-dose radiotherapy. Patients who received more radical radiation regimens may be those with more favorable ECOG PS and disease state. Another limitation was relatively small number of included cases in the final analysis. Large-scale prospective study is still needed to investigate the efficacy of SBRT for EGFR wild-type oligometastatic NSCLC patients. Additionally, this retrospective study performed without a control arm.

\section{Conclusions}

In summary, the current study indicated patients with EGFR wild-type NSCLC and oligometastatic disease who received SBRT delivering to primary tumor and all metastases could be treated safely and improved rates of OS and PFS. Patients with favorable ECOG PS were able to tolerate more aggressive radiation regimens (BED10 $\geq 100$ Gy) and had better outcomes. Further large-scale prospective randomized trials are still needed to define the role of SBRT in patients with EGFR wild-type NSCLCs and limited metastatic burden.

\section{Acknowledgments}

Funding: None.

\section{Footnote}

Reporting Checklist: The authors have completed the STROBE reporting checklist. Available at http://dx.doi. org/10.21037/tcr-20-2772

Data Sharing Statement: Available at http://dx.doi. org/10.21037/tcr-20-2772

Conflicts of Interest: All authors have completed the ICMJE uniform disclosure form (available at http://dx.doi. org/10.21037/tcr-20-2772). The authors have no conflicts of interest to declare.

Ethical Statement: The authors are accountable for all 
aspects of the work in ensuring that questions related to the accuracy or integrity of any part of the work are appropriately investigated and resolved. This retrospective study was conducted in accordance with the Declaration of Helsinki (as revised in 2013) and approved by the Ethics Committee of Air Force General Hospital (NO.: BJLNYY-\#2020009). The patients were included in this study only after obtaining orally or written informed consent.

Open Access Statement: This is an Open Access article distributed in accordance with the Creative Commons Attribution-NonCommercial-NoDerivs 4.0 International License (CC BY-NC-ND 4.0), which permits the noncommercial replication and distribution of the article with the strict proviso that no changes or edits are made and the original work is properly cited (including links to both the formal publication through the relevant DOI and the license). See: https://creativecommons.org/licenses/by-nc-nd/4.0/.

\section{References}

1. Torre LA, Bray F, Siegel RL, et al. Global cancer statistics, 2012. CA Cancer J Clin 2015;65:87-108.

2. Siegel RL, Miller KD, Jemal A: Cancer statistics, 2019. CA Cancer J Clin 2019;69:7-34.

3. Morgensztern D, Ng SH, Gao F, et al. Trends in stage distribution for patients with non-small cell lung cancer: a National Cancer Database survey. J Thorac Oncol 2010;5:29-33.

4. Ouyang WW, Su SF, Ma Z, et al. Prognosis of non-small cell lung cancer patients with bone oligometastases treated concurrently with thoracic three-dimensional radiotherapy and chemotherapy. Radiat Oncol 2014;9:147.

5. Mehta N, Mauer AM, Hellman S, et al. Analysis of further disease progression in metastatic non-small cell lung cancer: implications for locoregional treatment. Int J Oncol 2004;25:1677-83.

6. Weichselbaum RR, Hellman S. Oligometastases revisited. Nat Rev Clin Oncol 2011;8:378-82.

7. Hellman S, Weichselbaum RR. Oligometastases. J Clin Oncol 1995;13:8-10.

8. Planchard D, Popat S, Kerr K, et al. Metastatic non-small cell lung cancer: ESMO Clinical Practice Guidelines for diagnosis, treatment and follow-up. Ann Oncol 2018;29:iv192-iv237. Erratum in: Ann Oncol. 2019 May;30(5):863-870. doi: 10.1093/annonc/mdy474. Epub 2019 Dec 4.
9. Lopez Guerra JL, Gomez D, Zhuang Y, et al. Prognostic impact of radiation therapy to the primary tumor in patients with non-small cell lung cancer and oligometastasis at diagnosis. Int J Radiat Oncol Biol Phys 2012;84:e61-7.

10. Kim C, Hoang CD, Kesarwala AH, et al. Role of Local Ablative Therapy in Patients with Oligometastatic and Oligoprogressive Non-Small Cell Lung Cancer. J Thorac Oncol 2017;12:179-93.

11. Scagliotti GV, Parikh P, von Pawel J, et al. Phase III study comparing cisplatin plus gemcitabine with cisplatin plus pemetrexed in chemotherapy-naive patients with advanced-stage non-small-cell lung cancer. J Clin Oncol 2008;26:3543-51.

12. Weickhardt AJ, Scheier B, Burke JM, et al. Local ablative therapy of oligoprogressive disease prolongs disease control by tyrosine kinase inhibitors in oncogene-addicted non-small-cell lung cancer. J Thorac Oncol 2012;7:1807-14.

13. Jiang T, Chu Q, Wang H, et al. EGFR-TKIs plus local therapy demonstrated survival benefit than EGFRTKIs alone in EGFR-mutant NSCLC patients with oligometastatic or oligoprogressive liver metastases. Int J Cancer 2019;144:2605-12.

14. Sheu T, Heymach JV, Swisher SG, et al. Propensity scorematched analysis of comprehensive local therapy for oligometastatic non-small cell lung cancer that did not progress after front-line chemotherapy. Int $\mathrm{J}$ Radiat Oncol Biol Phys 2014;90:850-7.

15. Ouyang W, Yu J, Nuerjiang S, et al. Stereotactic body radiotherapy improves the survival of patients with oligometastatic non-small cell lung cancer. Cancer Med 2019;8:4605-14.

16. References National Cancer Institute Guidelines for Investigators: Common Terminology Criteria for Adverse Events (CTCAE) version 5.0. 2017. Available online: https://ctep.cancer.gov/protocolDevelopment/electronic_ applications/ctc.htm

17. Loriot $Y$, Soria JC, Le Chevalier T. Expanding role of chemotherapy in lung cancer. Ann Oncol 2006;17 Suppl 10:x101-x107.

18. Gomez DR, Blumenschein GR Jr, Lee JJ, et al. Local consolidative therapy versus maintenance therapy or observation for patients with oligometastatic non-smallcell lung cancer without progression after first-line systemic therapy: a multicentre, randomised, controlled, phase 2 study. Lancet Oncol 2016;17:1672-82.

19. De Ruysscher D, Wanders R, van Baardwijk A, et al. 
Radical treatment of non-small-cell lung cancer patients with synchronous oligometastases: long-term results of a prospective phase II trial (Nct01282450). J Thorac Oncol 2012;7:1547-55.

20. Agolli L, Valeriani M, Nicosia L, et al. Stereotactic Ablative Body Radiotherapy (SABR) in Pulmonary Oligometastatic/Oligorecurrent Non-small Cell Lung Cancer Patients: A New Therapeutic Approach. Anticancer Res 2015;35:6239-45.

21. Janssen S, Käsmann L, Rudat V, et al. Stereotactic Body Radiotherapy Provides Excellent Long-Term Local Control of Very Few Lung Metastases. In Vivo 2016;30:155-7.

22. Rusthoven KE, Hammerman SF, Kavanagh BD, et al. Is there a role for consolidative stereotactic body radiation therapy following first-line systemic therapy for metastatic lung cancer? A patterns-of-failure analysis. Acta Oncol 2009;48:578-83.

23. Rosell R, Carcereny E, Gervais R, et al. Erlotinib versus standard chemotherapy as first-line treatment for European patients with advanced EGFR mutation-positive non-small-cell lung cancer (EURTAC): a multicentre, open-label, randomised phase 3 trial. Lancet Oncol 2012;13:239-46.

24. $\mathrm{Wu} \mathrm{YL,} \mathrm{Zhou} \mathrm{C,} \mathrm{Hu} \mathrm{CP,} \mathrm{et} \mathrm{al.} \mathrm{Afatinib} \mathrm{versus} \mathrm{cisplatin}$ plus gemcitabine for first-line treatment of Asian patients with advanced non-small-cell lung cancer harbouring

Cite this article as: $\mathrm{Hu} \mathrm{X}, \mathrm{Li} \mathrm{H}$, Liu H, Liu Z, Xia T, Zhang J, Wang Y. Assessing efficacy and safety of stereotactic body radiation therapy for oligometastatic non-small cell lung cancer with epidermal growth factor receptor (EGFR) wild type. Transl Cancer Res 2021;10(1):184-194. doi: 10.21037/tcr-20-2772
EGFR mutations (LUX-Lung 6): an open-label, randomised phase 3 trial. Lancet Oncol 2014;15:213-22.

25. Xu Q, Zhou F, Liu H, et al. Consolidative Local Ablative Therapy Improves the Survival of Patients with Synchronous Oligometastatic NSCLC Harboring EGFR Activating Mutation Treated with First-Line EGFR-TKIs. J Thorac Oncol 2018;13:1383-92.

26. Nicosia L, Figlia V, Mazzola R, et al. Repeated stereotactic radiosurgery (SRS) using a non-coplanar mono-isocenter (HyperArc ${ }^{\mathrm{TM}}$ ) technique versus upfront whole-brain radiotherapy (WBRT): a matched-pair analysis. Clin Exp Metastasis 2020;37:77-83.

27. Widder J, Klinkenberg TJ, Ubbels JF, et al. Pulmonary oligometastases: metastasectomy or stereotactic ablative radiotherapy? Radiother Oncol 2013;107:409-13.

28. Nicosia L, Cuccia F, Mazzola R, et al. Stereotactic body radiotherapy (SBRT) can delay polymetastatic conversion in patients affected by liver oligometastases. J Cancer Res Clin Oncol 2020;146:2351-8.

29. Greco C, Pares O, Pimentel N, et al. PhenotypeOriented Ablation of Oligometastatic Cancer with Single Dose Radiation Therapy. Int J Radiat Oncol Biol Phys 2019;104:593-603.

30. Iyengar P, Wardak Z, Gerber DE, et al. Consolidative Radiotherapy for Limited Metastatic Non-Small-Cell Lung Cancer: A Phase 2 Randomized Clinical Trial. JAMA Oncol 2018;4:e173501. 


\section{Supplementary}

Table S1 Acute and long-term toxicities according to CTCAEv5.0

\begin{tabular}{lccc}
\hline CTCAEv5.0 & Grade 1, N (\%) & Grade 2, N (\%) & Grade 3, N (\%) \\
\hline Acute toxicities & $3(7.5)$ & $2(5.0)$ & 0 \\
Dermatitis & $7(17.5)$ & $3(7.5)$ & $1(2.5)$ \\
Pneumonia & $5(12.5)$ & $2(5.0)$ & 0 \\
Anemia & $14(35.0)$ & $5(12.5)$ & $1(2.5)$ \\
Leukopenia & $10(25.0)$ & $3(7.5)$ & 0 \\
Thrombocytopenia & $4(10.0)$ & $1(2.5)$ & 0 \\
Esophagitis & & $2(5.0)$ & 0 \\
Long-term toxicities & $2(5.0)$ & 0 & 0 \\
Pulmonary fibrosis & $1(2.5)$ & & 0 \\
Pleural effusion & & & \\
\hline
\end{tabular}

(C) Translational Cancer Research. All rights reserved. 